\title{
Mathematical modeling of gas flow distribution process in the gas transportation system of Armenia
}

\author{
Narine Pirumyan*and Mihran Stakyan \\ National University of Architecture and Construction of Armenia, 0009, Yerevan, Republic of \\ Armenia
}

\begin{abstract}
The issues of enhancement the methods of calculation and design of the gas transportation system (GTS) in the Republic of Armenia and the Republic of Artsakh are considered, taking into account the analysis of loading modes and the peculiarities of the system individual nodes' operation. To calculate the pressure distribution at the nodal points and gas flows, the matrix method for determining the material balance equations for the linear-independent and nonlinear-independent contours of the gas pipeline circuit is used as a process mathematical model. On the energy conservation law basis, the balance capacities equation of gas supply sources and its consumption, distributed among the gas transmission network elements, is proposed. A mathematical model of the gas distribution process is obtained. Calculation methods that allow increasing the economic efficiency of GTS operation under optimal terms of GTS development are proposed.
\end{abstract}

\section{Introduction}

Armenia is among the countries that import consumed fuel and energy resources (FER). Analysis of the primary energy resources consumption structure gives an opportunity to state a high share of imports of fuel and energy resources: 90.6...94.0\%, while a significant part is natural gas. The efficiency of using gas in comparison with other types of fuel is established on the basis of comparing the cost options of commercial products obtained as a result of using gas, or other types of fuel, provided that they are interchangeable. The principle is determined by the need to reduce production costs - to ensure the conditions for the profitability of enterprises with the production of competitive products. Minimization of costs is achieved by a step-by-step search of options - different combinations of used fuels (gas, fuel oil, coal).

During recent years natural gas began to occupy an increasingly significant position in the market, and its gas transportation system (GTS) is the most developed among the CIS member states. The gas supply system as a whole is managed by Gazprom Armenia CJSC and Energosystems CJSC of the Republic of Artsakh. As of 01.06.2019, the total length of the main gas pipelines in single-line terms is more than $1760 \mathrm{~km}$. The GTS of these

\footnotetext{
${ }^{*}$ Corresponding author: pirumyannarine@gmail.com
} 
republics as a whole is a network of main and looped distribution gas pipelines of a multithread structure with different pipe diameters, designed for operating pressure up to 5.5 $M P a$.

External gas supplies generally come from two sources: the Russian Federation and the Islamic Republic of Iran. To increase gas supply efficiency, it is proposed to build an additional section of the main gas pipeline, thanks to which it becomes possible to loop the two GTS into a single gas transmission system, which will permit the use of gas from the third source-the underground gas storage station of Armenia (SPCG) in Abovyan for all the regions of the Republic of Armenia and the Republic of Artsakh, as well as the reverse of Iranian natural gas in case of force majeure situations in the supply of Russian natural gas through the GTS of Georgia.

To carry out this most important activity, which ensures the energy security of the republic, it is necessary to implement the following work volume:

1. Geodetic survey and selection of the route of the new gas pipeline branch, determination of the volume of construction and ground works,

2. Computation and design of the gas pipeline branch under construction,

3. Execution of construction and installation works,

4. Testing and commissioning of a new branch of the gas pipeline.

Points 1 and 2 of these work stages can be performed simultaneously and contemporary methods of calculation and computer aided design using software can be used for the point 2.

\section{Calculation method}

GTS belongs to the class of large technical systems that have a complex internal structure, the presence of a large number of elements, as well as internal and external connections, continuously developing in space and time. The theory fundamentals of large energy systems, their classification, properties, and tasks were formulated in [1] and developed specifically for the pipeline systems [2-5]. The creation of large GTS, which are a set of complex subsystems (production, transport, storage and consumption of gas) and which have new properties resulting from their combination, imposes new requirements on the development of the theory and methods of analysis and synthesis of such systems used for the design and management of GTS modes. Therefore, the methodological problems of analysis and synthesis of modern large GTS are currently very relevant.

Calculation, design and management of complex GTS, which have acquired an important scientific, technical, industrial and socio-economic significance, are extremely complicated and can be successfully implemented in the development of methodological foundations, new principles, effective methods and the creation of computer aided design (CAD) systems for designing GTS. These systems perform all the basic operations related to the transformation, ordering and control of the source data, the formation of appropriate mathematical models, the organization of the computational process, the multivariate of calculations and the output of results in a convenient form. In this regard, the role of effective mathematical methods, models, algorithms and programs targeted to improving the increase defficiency of mathematical and CAD software for GTS.

At present, there are significant advances in the development of models describing flow distribution in hydraulic circuits with concentrated parameters, as well as algorithms and software for calculating complex pipeline and other hydraulic systems, which are successfully used in engineering calculations in practice [2, 3, 6, 7]. With the application of the flows and circuits energy theory main terms, new opportunities for research in many areas of engineering and communication systems have emerged, which has a reason for considering both general research methods and individual computational mathematical 
techniques from a new perspective. At the same time, the issues of developing theoretical methods for the analysis and synthesis of GTS also considered in a new way $[2,3,7,8]$.

GTS circuits have their own specifics and differ from the electrical circuits of the power system (except for the nonlinear dependencies of the state parameters) in that they:

- there are only longitudinal elements (pipelines and compressor stations-CS) and no transverse elements and contours;

- the inputs (gas sources) and outputs (gas consumers) are carried out with only one available clamp and it can be assumed that an ideal gas flow source is connected (similar to an ideal current source of an electric circuit);

- there is a CS element, which, with a constant gas flow in the section, sharply raises the gas pressure at its outlet.

These distinctive peculiarities led to the need to modify the structure of the energy equations, namely: the terms of the capacities of gas sources and consumers, since they do not constitute branches, are derived from the total sum of the terms for the branches of the contours and added as a separate sum for the nodes of the GTS with appropriate consideration of their signs.

In the case of steady gas movement in the GTS, the pressure distribution at the nodal points and gas flows on its branches is described with a certain accuracy by linear and nonlinear functions, which are the equations of state of the GTS and in some specific cases can be used with different forms of writing. The most appropriate of them is determined by the nature of the problem to be solved [9-14]. However, the application of these methods and computational programs for GTS with several hundred nodes and branches requires very time-consuming programming, reliability and convergence of the iterative calculation process.

A GTS chain of a random structure consisting of $S$ nodes, $n$ branches, and $L$ linearly independent contours is considered. The equation of the material balance in each node of the system has the form $\sum_{i=1}^{n} A_{j i} Q_{i}=q_{j}$, which in matrix form is represented as

$$
\left[A_{j i}\right] Q_{i}=q_{j} \text { or } A_{n} Q=q, \quad j=1,2, \cdots, s, i=1,2, \cdots, n
$$

where $Q_{i}$ is the flow of the transported gas from the $i$ branch; $q=q_{j}$ is the vector of gas withdrawals (inflows) from the system (to the system) in the the $j$-th node; $A_{n}=\left[A_{j i}\right]$ shows the complete incident matrix, i.e., the matrix of branch connections at nodes, the number of rows of which is equal to the number of the graphpeaks $S$, and the number of columns is equal to the number of edges $n$. The elements of this matrix can take one of three values $A_{j i}=1,=-1$, if the node, respectively, is the start and end of the branch $i$, $A_{j i}=0$, if the node $j$ does not belong to the branch $i$. Since in the chain graph, each branch connects two nodes, and the flow of this branch comes from one node and enters another node, the gas flow in each branch is included only in two equations of the system (1). Therefore, each column of the $A_{j i}$ matrix can have only one positive and negative "ones", and the remaining elements are "zeros". Therefore, the sum of all the rows of this matrix (by columns) should give a zero (row) matrix:

$$
n_{t} A_{n}=0
$$

where $n_{t}$ is a single row of $s \times 1$ dimension. 
If to select (2) in the row relevant to the balancing node, and take its number as last one, then the expression (2) is written in the following form:

$$
\left[n_{t}: 1\right]\left[\frac{A}{A_{b}}\right]=0, \text { where } A_{b}=-n_{t} A,
$$

where $A, A_{b}$ are respectively, the incident matrices for the schemes without a balancing and with a balancing node; $n_{t}$ is a single row of dimension $(s-1) x 1$. As a result of subtracting the last equation from (1), we get a system of (s-1) linearly independent equations:

$$
\sum_{i=1}^{n} A_{j i} Q_{i}=q_{j}, \quad j=1,2, \cdots,(s-1)
$$

which in matrix form can be represented as

$$
\left[A_{j i}\right] Q_{i}=q_{j} \text { or } A Q=q, j=1,2, \cdots,(s-1), \quad i=1,2, \cdots, n
$$

The nonlinear equations from $[7,9]$

$$
P_{j}^{2}-P_{k}^{2}=K_{i} Q_{i}^{2}, a_{i} P_{j}^{2}-P_{k}^{2}=b_{i} Q_{i}^{2}
$$

for a given structure of the GTS together can be represented as

$$
\sum_{j=1}^{s} \bar{A}_{j i}^{t} P_{j}^{2}=\sum_{j=1}^{s} \bar{A}_{i j} P_{j}^{2}=\bar{K}_{i} Q_{i}^{2}
$$

where $P_{j}, P_{k}$ define the pressure in the input and output $\mathrm{CS} ; \bar{A}_{j i}$ shows the elements of a complete rectangular matrix of connections of nodes and branches for the GTS scheme with $\mathrm{CS}$, which uniquely describes the structure of this scheme and the orientation of its branches. Here $\bar{A}_{j i}=1$, if the node $j$ is the initial for a branch without $\mathrm{CS} ; \bar{A}_{j i}=0$, if the node $j$ is the initial for a branch $i$ without $\mathrm{CS}, \bar{A}_{j i}=0$, if the node $j$ is final for the branches $I$ with CS and without CS (i.e., the branch $i$ with CS and without CS is oriented in the direction of the node $j$ ); $\bar{A}_{j i}=0$ if the node $j$ does not belong to the branch $i$.

The system (7) in matrix form is represented as

$$
\bar{A}^{t} P^{2}=\left[E_{n} \bar{K}\right] Q^{2} \text { or }\left[\bar{A}_{i j}\right] P_{j}^{2}=\left[E_{n} \bar{K}_{i}\right] Q_{i}^{2}, \quad j=1,2, \cdots, s, i=1,2, \cdots, n,
$$

where $E_{n}$ is the single matrix of $n^{2}$ dimension, $\bar{A}^{t}$ is the transposed matrix with respect to $\bar{A}_{j i}, \bar{K}_{i}$ are the coefficient, which at the operating pressure of $P_{i}>0.3 M P a$ for stationary and isothermal conditions is determined by the formula [8]

$$
K_{i}=\frac{\Delta T_{\text {ave }} Z_{\text {ave }} L_{i}}{1.67^{2} \cdot 10^{-12} \cdot D_{i}^{5.2}}
$$


where $\Delta=0.6$ is the relative density of the gas, $T_{a v e}=296 \mathrm{~K}$ is the average temperature of the gas at the gas pipe section, $Z_{\text {ave }}$ is a gas compressibility factor at medium pressure data and temperature at the section, $L_{i}, D_{i}$ show the equivalent length $(\mathrm{km})$ and diameter $i$-th of the gas pipe section $(\mathrm{mm})$.

The equation (6) can be used for the complex sections of the pipeline with parallel threads or series-connected subsections (segments) of different geometry.

Another important component of the GTS is the CS, which ensures the flow of transported gas through gas pipelines and brings energy to the system. The CS in the hydraulic circuit of the GTS is a non-linear element that sharply increases the outlet pressure at a constant value of the gas flow passing through it.

The form of writing the equation of the CS and the gas pipeline section (6) is convenient for their joint solution and analysis of the GTS modes. However, the presence of equivalent parameters $a_{i}$ and $b_{i}$ in the expression (6) makes it much more difficult to represent the equations in a compact canonical form of notation.

In many works, in particular [7,14], these expressions in the form of the pressure drops balance equation along the contour are presented using implicit functions, in which all the GTS state variables do not explicitly participate. To overcome this difficulty, the equations of $\mathrm{COP}(6)$ are presented in a different form of writing:

$$
P_{j}^{2}-P_{k}^{2}=b_{i} Q_{i}^{2}-h_{i}
$$

where $h_{i}=\left(a_{i}-1\right) P_{j}^{2}$ is the additional summand of the equation (a.s.e.) of the $i$-th section of the CS, $i \in l_{2}$. Then, for a GTS chain of an arbitrary structure, equation (10) can be represented by the matrix equation

$$
A_{n}^{t} P^{2}=\left[E_{n} \bar{K}\right] Q^{2}-h \quad \text { or } \quad\left[A_{i j}\right] P_{j}^{2}=\left[E_{n} \bar{K}_{i}\right] Q_{i}^{2}-h_{i}
$$

where $h=h_{i}=\left(a_{i}-1\right) P_{j}^{2}$ is the vector of a. s. e. COP $1 \times n ; ; E_{n}$ is the single matrix of $n^{2}$ dimension.

The equations of state for linearly independent contours are compiled, for which a rectangular matrix of branch connections in independent contours is introduced

$$
B=\left[B_{l i}\right] l=1,2, \ldots, L, \quad i=1,2, \ldots, n
$$

the number of rows of which is equal to the chain graph independent contours number, $L$ and the number of columns is equal to the number of branches $n$.

The balance of the drop (losses) of the square of the pressure $\Delta P_{i}=P_{j}^{2}-P_{k}^{2}$ over the closed loops of the circuit is formulated as follows: the algebraic sum of the drops $\Delta P_{i}$ on the branches of the circuit is zero, i.e.,

$$
B \cdot \Delta P=0 \text { or }\left[B_{l i}\right] \cdot \Delta P_{i}=0, \quad l=1,2, \ldots, L, \quad i=1,2, \ldots, n
$$

Using the full incident matrix, the drop of the $\Delta P_{i}$ on the branches of the circuitry can be determined from the nodal pressures. Since each column of the matrix $A_{n}$ has one positive "one" at the place of the initial peak and one negative "one" at the final peak of the branch, 
it's sufficient to multiply the transposed matrix of connections $A_{n}^{t}=A_{i j}$ on the right by the column of the nodal pressures square $P^{2}=P_{j}^{2}$ to obtain a column of pressures square differences at the ends of each branch, i.e., drop $\Delta P$ on the branches:

$$
\Delta P=A_{n}^{t} P^{2} \text { or } \Delta P_{i}=\left[A_{i j}\right] P_{j}^{2} .
$$

Considering that $\Delta P=\Delta P_{i}$, connected and solved (11), (13), and (14), we get the expression

$$
\begin{gathered}
{\left[B_{l i}\right]\left[A_{i j}\right] P_{j}^{2}=\left[B_{l i}\right]\left[E_{n} \bar{K}_{i}\right] Q_{i}^{2}-h_{l}=0 \text { or }} \\
{\left[B_{l i}\right]\left[A_{i j}\right] P_{j}^{2}=\left[B_{l i}\right]\left[E_{n} \bar{K}_{i}\right] Q_{i}^{2}-h_{l}=0,}
\end{gathered}
$$

where $h_{l}=\left[B_{l j}\right] h_{i}$ is a column of contour a.s.e. of CS, representing the algebraic sums of a.s.e. of the $i$ branches of CS included in each independent contour.

The vector $P$ components included in (15) are the nodal pressures of all the $s$ nodes of the GTS circuit (including the balancing one). Often, it is advisable to determine them relative to the balancing node, i.e., as the difference of the squares from each of the independent nodes of the GTS circuit with respect to the square of the pressure of the balancing node. These values differ from the squares of the nodal pressures $P_{j}(j=1,2, \ldots, s)$ included in (15) by the same magnitude - the balancing node $P_{b}^{2}$ pressures square (in this case, the balancing node is assumed to be the last by number, i.e., $P_{s}=P_{b}$ ). To do this, the complete incident matrix included in (15) and the nodal pressure vector can be divided into blocks and sub-vectors as follows:

$$
A_{n}=\left[\frac{A}{-n_{t} A}\right] ; \quad P=\left[\frac{P_{j}}{P_{s}}\right], \quad j=1,2, \cdots,(s-1) .
$$

Then the expression (14) for the squared pressures differences on the GTS scheme branches can be represented as:

$$
\Delta P=A_{n}^{t} P=\left[A^{t}-A^{t} n\right]\left[\frac{P_{j}^{2}}{P_{s}^{2}}\right]=A^{t}\left(P_{j}^{2}-n P_{s}^{2}\right), \quad j=1,2, \cdots,(s-1)
$$

where $n$ is a singlecolumn of dimension $1 \mathrm{x}(\mathrm{s}-1)$.

Jointly solving (13) and (16), we get a matrix equation in a different form of writing

$$
\begin{gathered}
B A^{t}\left(P_{j}^{2}-n P_{s}^{2}\right)=B\left[E_{n} \bar{K}_{i}\right] Q^{2}-h_{l}=0 \quad \text { or } \\
{\left[B_{l i}\right]\left[A_{i j}\right]\left(P_{j}^{2}-P_{s}^{2}\right)=\left[B_{l i}\right]\left[E_{n} \bar{K}_{i}\right] Q_{i}^{2}-h_{l}=0}
\end{gathered}
$$




\section{Results and discussion}

To form the equation of GTS state, according to (5) and (17), it is necessary preliminarily to determine the matrix of connections $A$ and $B$, which in analytical form definitely display the configuration of the circuit. The matrix $A$ contains comprehensive information about the circuit scheme, and it is possible to uniquely restore the corresponding scheme configuration.

In general, the matrix $B$ does not contain complete information about the structure of the GTS scheme under consideration, since the open parts of the scheme are not reflected in it. The difficulties associated with the formation of the matrix $B$ can be avoided if to take into account the fact that the matrix $A$ contains comprehensive information about the structure of the GTS scheme, including the information necessary for the compilation of the matrix $B$. To implement this opportunity, it is necessary to establish an analytical relationship linking the $A$ and $B$ matrices.

Since the equation (17) is valid for any nodal pressure vector and $P_{j} \neq 0, P_{s} \neq 0$, therefore, it must always be

$$
B A^{t}=\left[B_{l i}\right]\left[A_{i j}\right]=0
$$

Expression (18) displays the general topological property of the GTS chain graph. Knowing the matrix $A$, it is possible to find the matrix $B$ using the relation (18) and the elements of graph theory.

The mathematical model of the CS (6) as a whole is obtained by synthesizing the equations of individual gas pumping units obtained by approximating the graphical characteristics taking into account the gas pressure losses in the CS communications [15]. New physical values for GTS have also been introduced, for example, capacity (or quasicapacity) in the form of the product of gas pressure and gas flow for gas pipeline sections and sections with CS. If $q_{j}>0$, then the product $S_{j}=P_{j} q_{j}$ is the power of the gas source that supplies the system with gas through the $j$-th node. At the flow dimension $|q|=$ million $\mathrm{m}^{3}$ /day and the pressure of the $|P|=0.1 \mathrm{MPa}$ product $S_{j}$ has the dimension of capacity, i.e.

$$
|S|=\text { million } \mathrm{m}^{3} \mathrm{~kg} / \text { day. } \mathrm{cm}^{2}=1.14 M W
$$

If the selection from the system of the gas consumer from the $j$-th node is $q_{j}<0$, then the product $S_{j}=P_{j} q_{j}$ is the negative power of the gas consumer. For underground gas storage station (UGSS), if it operates in the injection mode, the product is a negative value representing the capacity of the UGSS as a gas consumer. In the gas extraction mode from the UGSS, this product is a positive value, which represents the power of the UGSS as a gas source. 


\section{Conclusions}

In a complex chain consisting of $s$ nodes and $n$ branches, nodal pressures $P_{j}(j=1,2, \cdots, s)$, gas flows in the $Q_{i}(i=1,2, \cdots, n)$ branches, and external flows (inflows and withdrawals) of gas $q_{j}(j=1,2, \cdots, s)$ are related by the equations (4), (17). However, for the same chain, only one equation that connects these parameters together can be created. Taking into account the distinctive features of the GTS circuits, based on the law of energy conservation for a given GTS circuit, one can write the balance of the gas supply sources' capacities, gas consumers and power loss (energy dissipation) in the GTS network in the form

$$
\sum_{j=1}^{s} P_{j} q_{j}-\sum_{i=1}^{n} \Pi_{i} Q_{i}=0
$$

This equation shows that the sum of the pressures $P_{j}$ products and external gas flows $q_{j}$ of all $\bar{s}$ nodes of the GTS chain directed graph minus the sum of the pressure drop $\Pi_{i}$ all $\bar{n}$ branches' products on the relevant gas flows $Q_{i}$, is zero.

The expression (19) is the energy equation of the GTS chain power balance and simultaneously expresses the law of energy conservation for the same chain. The physical meaning of this equation is explained as follows: at each moment of time, the capacity(energy) that is introduced into the GTS chain through its inputs and through the $\mathrm{CS}$ is consumed by gas consumers through its outputs and distributed among the chain elements (gas pipelines) in the form of dissipation, i.e., power loss.

GTS is characterized by the interconnectedness of the parameters and the variety of modes of its operation. The problem of controlling its complex modes and computer aided design is becoming more and more relevant every year. The solution to this problem requires the development of qualitatively new approaches to the analysis and synthesis of large systems with the most rational use of the capabilities of software tools [14,16-20].

This work has been carried out in the frame of "Preservation and development of the research laboratory of natural-mathematical modelling of construction tasks" and "Preservation and development of the research laboratory of construction and urban economy" programmesffinanced by Science Committee of Republic of Armenia.

\section{References}

1. L. A. Melentyev, Bulletin of NA SSSR. Energy and Transport 3, 3-9 (1980).

2. A. A. Ionin, Gas supply (Moscow,1989)

3. A. P. Merenkov, V. Ya. Khosilev, Theory of hydraulic media (1985)

4. V. S. Pankratov, A.V. Dubinsky, B. I. Siperstein et al, Software and technical means of automated control systems and experience of their operation in gas transport associations (Moscow, 1986)

5. M. G. Sukharev, E. R. Stavrovsky, V. E. Bryanskikh, Optimal development of gas supply systems Moscow (Moscow, 1981).

6. S. G. Akopyan, Calculation of the flow distribution of the stationary regime of the gas transmission system, Proceedings: The problem of improving and developing 
progressive technical and economic norms and standards in the gas industry, 81-91 (Moscow, 1982)

7. V. S. Pankratov, A.V. Dubinsky, Information and computing systems in the dispatching control of gas pipelines (Leningrad, 1988)

8. E. L. Volsky, A. I. Garlyauskas, S. V. Gerchikov, Reliability and optimal redundancy of gas fields and main gas pipelines (Moscow, 1980)

9. N. V. Pirumyan, H. A. Khazaryan, Improvement of methods of calculation and design of the gas transmission system of Armenia, Proceedingsof conf.: "Logistics, Transport, Ecology-2018", 126-132 (Yerevan, 2018)

10. N. V. Pirumyan, H. A. Khazaryan, M. G. Stukyan, Study of work reliability of gas subsurface storage by the method of finite elements, Proceedings of the 10th Int. Conf. on Contemporary Problems of Architecture and Construction, 76-78 (Beijing, 2018)

11. H.A. Ghazaryan, S.S. Chibukhchyan, M.G. Stakyan, Increasing reliability of gastransportation system (GTS) by constructions and vibration stability criteria, Proceedings of 8-th Inter. Conf. on Cont. Problems of Archit. and Constr., 168-170 (Yerevan, 2016).

12. M. G.Stakyan, A. A. Kazaryan, Yu. A. Kazaryan, Bulletin of NUACA 2 (51), 74-77 (2016).

13. N. V. Pirumyan, A. A. Kazaryan, M. G. Stukyan, Scientific Papers of NUACA 1 (64), 130-136 (2017).

14. N. V. Pirumyan, M. G. Stukyan, IOP Conf. Se.: Materials Sci. Eng. 698, 066022 (2019). https://iopscience.iop.org/article/10.1088/1757-899X/698/6/066022

15. R. Ya. Berman, V. S. Pankratov, Automation of control systems for main gas pipelines. (Nedra, Leningrad, 1978).

16. N. V. Pirumyan, M.G. Stakyan, G.Sh. Galstyan, Key Engineering Materials 828, 115120 (2020). https://doi.org/10.4028/www.scientific.net/kem.828.115

17. N. V. Pirumyan, M.G. Stakyan, G.Sh. Galstyan, Key Engineering Materials 828, 121128 (2020). https://doi.org/10.4028/www.scientific.net/kem.828.121

18. N. V. Pirumyan M.G. Stakyan, IOP Conf. Ser.: Mater. Sci. Eng. 913, 022035 (2020). https://iopscience.iop.org/article/10.1088/1757-899X/913/2/022035/pdf

19. N. V. Pirumyan, M.G. Stakyan, IOP Conf. Se.: Materials Sci.Eng. 698, 022077 (2019). https://iopscience.iop.org/article/10.1088/1757-899X/698/2/022077/pdf

20. N. V. Pirumyan, M.G. Stakyan, IOP Conf. Ser.: Mater. Sci. Eng. 913, 022006 (2020). https://iopscience.iop.org/article/10.1088/1757-899X/913/2/022006/pdf 